CERN-TH/98-09

hep-th/9801064

\title{
Effectiveness of One-Dimensional Gas Models for Black Holes
}

\author{
A. Ghosh \\ CERN \\ Theory Division \\ CH-1211 Geneva 23, Switzerland
}

\begin{abstract}
A one-dimensional gas model has been constructed and is shown to provide correct expressions for entropies for extremal and near-extremal BTZ black holes. Recently suggested boosting of black strings is also used to compute the entropy for the Schwarzschild black hole from this gas model.
\end{abstract}

CERN-TH/98-09

January, 1998 


\section{Introduction}

In this note we attempt to propose a free one-dimensional bosonic gas model to describe the relevant degrees of freedom of a black hole, based on some previous attempts, which addressed the cases of some specific black holes [1,2]. We show that it is possible to identify this way of counting states with the counting procedure in a conformal field theory when mappings are prescribed between the appropriate parameters in a conformal field theory and the gas model. Note that no supersymmetry has been invoked in this model.

BTZ black holes in $2+1$ dimensions provide a good example of attempting to understand the black hole entropy by counting the microstates near its horizon [3]. Subsequently exact conformal field theories have been constructed for this case [4]. Recently a class of geometries have also been identified which share features with the BTZ case [5]. We show here that the extremal and near-extremal BTZ black holes can be adequately described by an appropriate one-dimensional gas of bosons by computing its entropy from the gas model. In some sense, it reconfirms all the previous calculations of the counting of states. The gas model cannot predict the location of the states for the black hole. We also describe the importance of introducing a ground state advocated by many authors $[4,5]$ in the context of constructing the conformal field theory description for BTZ black holes. In the context of the gas model, this will appear in a natural way and will provide a better starting point for its implementation. We will make use of this concept to make an improvement of the conventional gas model picture. The situation, however, can hardly be improved for (3+1)-dimensional Reissner-Nordstrom black holes or Schwarzschild black holes. We investigate this situation in some detail in section 5. We also pointed out the essential objections against the flat Minkowski space being treated as the possible 'ground state' for most of the asymptotically flat black holes in $3+1$ dimensions.

There have been many attempts [6] to provide a counting of states for the Schwarzschild black holes in various dimensions, including recently some from Mtheory. The key idea is to map the Schwarzschild black hole to extremal or nearextremal configurations from which the counting might be possible. One concrete realization of this mapping was provided by [7], where it is shown that black branes/strings give rise to Schwarzschild or charged dilatonic black holes when reduced to lower dimensions before or after boosting along some uncompactified direction. This mapping has been used to argue even the matching of entropies for these

two distinct classes of black holes [8]. Some counting has also been performed for seven-dimensional Schwarzschild black holes, by computing the entropy of a near- 
extremal three-brane obtained by this boosting and dualizations [9]. We provide such a boosting on a five-dimensional black string to map to a charged dilatonic black hole. Then a gas model computation of the entropy has been performed, which eventually predicts, by construction, the entropy of a Schwarzschild black hole. In this way in $3+1$ dimensions we found an exact agreement between the gas model and the Schwarzschild black hole entropy.

We first describe the model and its relation to conformal field theories in sections 2 and 3. In subsequent sections we apply it to various black holes in various dimensions. Starting with the $(2+1)$-dimensional BTZ in section 4 , we put forward an improved version of the model in section 5. Schwarzschild black holes in $3+1$ dimensions are discussed in section 6 .

\section{The gas model}

In this section I shall describe the model. Let us take a one-dimensional gas consisting of $N$ massless bosons in thermal equilibrium at temperature $T$. The space is a box of length $L$ with periodic boundary conditions at the ends. We have used physical units throughout and the Newton constant, according to our conveniences, is taken to have various values, which will be specified later on. The spectrum of the model consists of an infinite number of oscillators with energy eigenvalues

$$
E_{n}=n \omega, \quad n \in \mathbf{Z}, \omega=\frac{2 \pi}{L} .
$$

The free energy of this system is given by the partition function

$$
\begin{aligned}
\beta F & =\sum_{n} \log \left[1-\exp \left(-\beta E_{n}\right)\right] \\
& \simeq \int_{0}^{\infty} d n \log [1-\exp (-\beta \hbar n \omega)]=-\frac{\pi^{2}}{6 \beta \omega}
\end{aligned}
$$

i.e.

$$
F=-\frac{\pi T^{2} L}{12}
$$

All states being massless, there will be in general left-moving and right-moving modes for which we put indices $L$ and $R$. The expressions for free energy, entropy and total energy are the following

$$
F_{L, R}=-\frac{\pi}{12} L N_{L, R} T_{L, R}^{2}
$$




$$
\begin{aligned}
S_{L, R} & =-\partial F_{L, R} / \partial T_{L, R}=\frac{\pi}{6} L N_{L, R} T_{L, R} \\
E_{L, R} & =F_{L, R}+T_{L, R} S_{L, R}=\frac{\pi}{12} L N_{L, R} T_{L, R}^{2}
\end{aligned}
$$

with left (right) oscillators are in equilibrium at temperature $T_{L(R)}$. Combining left and right sectors, the total energy and momentum of the system will be given by

$$
\begin{aligned}
E & =E_{L}+E_{R}+E_{0} \\
P & =E_{L}-E_{R},
\end{aligned}
$$

where $E_{0}$ is the thermal ground state energy correction, which we have left out in (2.1). Throughout the calculation we shall keep the net momentum $P$ and ground state energy $E_{0}$ fixed for the system. The reason for doing this will be clarified when we come back to this point. The immediate consequences are

$$
d P=d E_{L}-d E_{R}=0, d E_{0}=0 \Rightarrow d E=2 d E_{L}=2 d E_{R}
$$

So the net temperature $T$ is given by the formula

$$
\frac{1}{T}=\frac{\partial S}{\partial E}=\frac{\partial S_{L}}{2 \partial E_{L}}+\frac{\partial S_{R}}{2 \partial E_{R}}=\frac{1}{2}\left(\frac{1}{T_{L}}+\frac{1}{T_{R}}\right) .
$$

These are all the formulas that we need for our present purpose. We shall apply this model to describe excitations of a generic black hole over a corresponding 'ground state'. The meaning and importance of choosing this 'ground state' will be clarified later. Our strategy will be the following: we shall identify the temperature and the energy of the gas with the corresponding objects for black holes (by energy of the black hole we mean its ADM energy). Then we shall show that the entropy of the gas model predicts exactly the entropy of the black hole. At this point we shall see how the entropy of the gas model compares with the exact conformal field theory(CFT) counting.

\section{Relation to CFT}

Here we discuss how the model described in the previous section relates to CFT. The basic motivation would be to try to identify this model with some conformal field theory whose left and right sectors are to be realized as the left and right oscillators of this model. First we define the central charge from the gas model to be $c=N_{L}=N_{R}$. Now let us take a primary state in the CFT at level $\left(h_{L}, h_{R}\right)$ and associate this with the quantum mechanical state of a black hole:

$$
\left.\left|h_{L}, h_{R}\right\rangle \equiv \mid \text { black hole }\right\rangle
$$


Clearly, the underlying assumption is that the quantum theory of gravity, in a restricted sense, is an exact CFT. This does not sound unnatural at present as many examples have already been constructed and 'verified' $[3,10]$. In CFT the degeneracy of this state (for $h_{L, R} \gg 1$ ) is known to be

$$
d\left(h_{L}, h_{R}, c\right) \simeq \exp \left[2 \pi \sqrt{\frac{c h_{L}}{6}}+2 \pi \sqrt{\frac{c h_{R}}{6}}\right] .
$$

So the Boltzmann entropy of this state is given by

$$
S_{B}=\log d\left(h_{L}, h_{R}, c\right) \simeq 2 \pi \sqrt{\frac{c h_{L}}{6}}+2 \pi \sqrt{\frac{c h_{R}}{6}} .
$$

Now for the gas model if we define the levels $h_{L}, h_{R}$ by

$$
P=2 \pi \frac{h_{L}-h_{R}}{L}
$$

then immediately we get

$$
S_{B}=\frac{\pi L}{6}\left[N_{L} T_{L}+N_{R} T_{R}\right]=S
$$

where we have used $c=N_{L}=N_{R}$. The implication of the above result is that whenever the gas model entropies would give rise to the correct expressions for the black hole entropies then there always exists a natural, underlying CFT that can also be used to describe the black hole states. In this note we shall give evidence of this implication only by computing black hole entropies. Of course, more evidence should be collected by computing other objects, such as emission and absorption cross sections of black holes. Note, however, that this identification of $S_{B}=S_{\text {gas }}$ is independent of whether the gas model is applied to black holes or not.

\section{Entropy of BTZ black holes}

Let us consider the case of the three-dimensional BTZ black hole for which the entropy has already been calculated, using exact conformal field theories $[3,4]$. Here we are doing this exercise just to provide more evidence to the parallelism of the gas model and CFTs. It is important to notice that this does not require the existence of supersymmetry in support of the correctness of the counting or validity of the formulation. We shall take up the case of the near-extremal BTZ black hole. The reason is that, for exact non-extremal BTZ black hole, the identifications of temperature 
and energy with those of the gas model do not give sufficient information about the model, so that we can compute the entropy. However, for the black hole near to its extremality some detailed structure of the gas model can be used to completely specify all the relevant parameters for computing its entropy from just the temperature and energy equations. In this section we shall first use the extremal black hole to fix some gas model parameters. In the next section we shall improve on this procedure to predict the near-extremal entropy from more elementary considerations.

Gravity in $2+1$ dimensions with a cosmological constant $\Lambda=-1 / l^{2}$, furnishes rotating black hole solutions (BTZ black hole) [11]

$$
d s^{2}=-f^{2} d t^{2}+f^{-2} d r^{2}+r^{2}\left(d \phi-\frac{J}{2 r^{2}} d t\right)^{2},
$$

where $f^{2}=-M+r^{2} / l^{2}+J^{2} / 4 r^{2}$. The two horizons, the temperature and the entropy of this black hole are given by

$$
\begin{aligned}
r_{ \pm}^{2} & =\frac{1}{2} M l^{2}\left[1 \pm\left(1-\frac{J^{2}}{M^{2} l^{2}}\right)^{1 / 2}\right] \\
T_{H} & =\frac{r_{+}^{2}-r_{-}^{2}}{2 \pi r_{+} l^{2}} \\
S_{H} & =4 \pi r_{+},
\end{aligned}
$$

where we have taken the Newton constant to be $G_{N}=1 / 8$. The extremal limit of this black hole amounts to identifying the two horizons $r_{+}=r_{-}$or $J=M l$.

Now let us use the extremal black hole parameters to restrict the gas model to some extent. We shall equate energy, temperature and entropy to determine some parameters of the gas model. For the extremal solution

$$
T_{H}=0, E_{H}=|J| / l, S_{H}=4 \pi \sqrt{J l / 2} .
$$

First, equating the temperatures one finds that there are two possibilities: a) both left and right temperatures are zero and b) one of the two is zero. But the possibility (a) can be discarded by considering the entropy. Let us take $T_{R}=0 \Rightarrow E_{R}=0$. So

$$
\begin{aligned}
\frac{J}{l} & =E_{0}+\frac{\pi L N_{L}}{12} T_{L}^{(0) 2} \\
4 \pi \sqrt{J l / 2} & =\frac{\pi L N_{L}}{6} T_{L}^{(0)},
\end{aligned}
$$

where the superscript (0) stands for objects in the extremal case. Equations (4.16) give rise to

$$
T_{L}^{(0)}=\frac{24}{N_{L} L} \sqrt{\frac{J l}{2}}
$$




$$
\begin{aligned}
E_{0} & =\frac{J}{l}-\frac{24 \pi J l}{N_{L} L} \\
P & =\frac{J}{l}-E_{0} .
\end{aligned}
$$

Now let us feed it to the near-extremal case for which $M=J / l+\epsilon, \epsilon \ll J / l$. The two horizons, the Hawking temperature and the entropy are given by

$$
\begin{aligned}
& r_{ \pm} \simeq \sqrt{\frac{J l}{2}}\left(1 \pm \sqrt{\frac{\epsilon l}{2 J}}+\frac{\epsilon l}{2 J}\right)+O\left(\epsilon^{3 / 2}\right) \\
& T_{H} \simeq \frac{1}{l \pi}\left(\sqrt{\epsilon}-\epsilon \sqrt{\frac{l}{2 J}}\right)+O\left(\epsilon^{3 / 2}\right) \\
& S_{H} \simeq 4 \pi \sqrt{\frac{J l}{2}}+2 \pi l \sqrt{\epsilon}+\pi l \epsilon \sqrt{\frac{l}{2 J}}+O\left(\epsilon^{3 / 2}\right) .
\end{aligned}
$$

In the gas model we shall keep $P$ and $E_{0}$ fixed (their values correspond to the BTZ class in the sense that they are fixed by the extremal solution alone; similar observations have been made recently for the case of dyonic black holes too [12]). This gives rise to the following expressions for $E_{L}, E_{R}$

$$
\begin{aligned}
E_{L}+E_{0} & =\frac{J}{l}+\frac{\epsilon}{2} \\
E_{R} & =\frac{\epsilon}{2},
\end{aligned}
$$

so that $E=E_{L}+E_{R}+E_{0}=J / l+\epsilon=M$ and $P=E_{L}-E_{R}=J / l-E_{0}$. From the above identifications we find $T_{L}$ and $T_{R}$ to be (using $E_{0}$ )

$$
\begin{aligned}
& T_{L} \simeq \frac{24}{L N_{L}} \sqrt{\frac{J l}{2}}+\frac{\epsilon}{4 \pi \sqrt{2 J l}}+O\left(\epsilon^{3 / 2}\right) \\
& T_{R}=\left(\frac{6 \epsilon}{\pi L N_{R}}\right)^{1 / 2} .
\end{aligned}
$$

So the net temperature of the gas is given by

$$
T=\frac{2 T_{L} T_{R}}{T_{L}+T_{R}} \simeq 2\left(\frac{6 \epsilon}{\pi L N_{R}}\right)^{1 / 2}-\frac{\epsilon N_{L}}{l \pi N_{R}} \sqrt{\frac{l}{2 J}}+O\left(\epsilon^{3 / 2}\right) .
$$

Comparing this temperature with $T_{H}$ the $\sqrt{\epsilon}$-terms give a value for $L N_{R}$ and the $\epsilon$-terms give a value for $L N_{L}$ :

$$
L N_{L}=L N_{R}=24 \pi l^{2} .
$$


If we put all these values in (2.15) and (2.17) then the entropy becomes

$$
S=\frac{\pi L}{6}\left(N_{L} T_{L}+N_{R} T_{R}\right)=S_{H}
$$

up to order $\epsilon$. At this point let me also mention the relation of these calculations

with [3]. It is natural to take $L=l$, the scale set by the cosmological constant. Then the central charge of the corresponding CFT is given by $c=24 \pi l$, in agreement with [3]. If we identify the levels from their definitions (3.11) and put them in (3.10), then using the above central charge the entropy formula in (4.18) is easily reproduced.

\section{A detour}

In this section we make an improvement of the naive implementation of the gas model made in the previous section. As an example, consider again the case of the BTZ black hole. Of course this detour follows the basic procedure adopted in the previous section, but we will see that this can reproduce the results obtained in section 4 more independently. The philosophy is the following: we shall first identify a suitable 'ground state' for the black hole and eventually build up our black hole over that ground state in a systematic way. The corresponding gas model description will follow automatically from this construction.

For BTZ let us start from the state described by the configuration $M=J=0$

$$
d s^{2}=-\frac{r^{2}}{l^{2}} d t^{2}+\frac{l^{2}}{r^{2}} d r^{2}+r^{2} d \phi^{2}
$$

The relevance of this 'ground state' is also described in $[4,5]$, where it is identified as the ground state in the Ramond sector of a super-CFT whereas the adS-space-time is identified as the ground state in the Neveu-Schwarz sector. The CFT relevant to the BTZ black hole is equivalent to the quantum theory of gravity built on the adS vacuum. We will not invoke any supersymmetry here and show, in the context of the gas model, that excitations over this ground state alone are adequate for describing the near-extremal BTZ configuration. For this state we have [4], $E_{H}=T_{H}=S_{H}=0$. From the particle model point of view, both $T_{L}, T_{R}$ should be vanishing as the entropy is zero in this case, from which we conclude that the ground state energy $E_{0}=0$. Also the important thing to notice is that for this configuration the left and right sectors of the particle model behave democratically. Now over this state we want to build up the extremal BTZ configuration. One way would be to throw an arbitrary amount of matter such that $M=J / L$ always holds. We know from our analysis 
in the previous section that for this configuration the particle model stops behaving democratically in its left and right sectors. The basic reason is that one cannot put both $T_{L}, T_{R}$ to zero, because the extremal black hole has a finite entropy. The right sector still remains at its ground state and the left sector picks up excitations (this was our convention in the earlier section). Then at once we can identify the energy and the entropy to be

$$
S=2 E_{L} / T_{L}, \quad \text { where } E_{L}=J / l
$$

We do not learn anything more than this as $T_{L}$ remains an arbitrary parameter. On the contrary, we will now see how all the information can be extracted if we relax the extremality condition a little bit. This is more natural from the point of view of how the extremal configurations are actually obtained: first throw an arbitrary amount of matter such that we do not care about the extremality holding exactly, but it is sufficient to restrict to a near-extremal configuration and let $\epsilon \rightarrow 0$ at the end of the process. So we let our temperatures be

$$
\begin{aligned}
& T_{R}=a_{1} \sqrt{\epsilon}+a_{2} \epsilon+\ldots \\
& T_{L}=T_{0}+b_{1} \sqrt{\epsilon}+b_{2} \epsilon+\ldots
\end{aligned}
$$

We will fix these unknown constant coefficients $T_{0}, a_{i}, b_{i}$ 's by comparing gas model temperature and energy with the corresponding objects for the black hole. The temperature identification immediately gives

$$
a_{1}=\frac{1}{2 \pi L}, \quad a_{2}=0, \quad T_{0}=\frac{1}{\pi l^{2}} \sqrt{\frac{J l}{2}}
$$

$a_{2}=0$ does not follow automatically from this, but it turns out to be zero by keeping $E_{0}=0$ fixed up to order $\epsilon$. We still use the basic decomposition equations (4.19) to fix the other constants (when $P, E_{0}$ are kept fixed):

$$
b_{1}=0, \quad b_{2}=\frac{1}{4 \pi \sqrt{2 J l}}, \quad L N_{L}=L N_{R}=24 \pi l^{2}
$$

So we have reproduced all the information about the gas model required to compute the entropy. Recall that here we did not make use of the energy, the temperature or the entropy of the extremal configuration explicitly. This is why this approach is more powerful.

When applied to other cases the situation is not so robust. One needs to fix a funny ground state energy $E_{0}$ by making use of the extremal configuration explicitly. Then 
we are back to the level of the naive gas model. The situation can be demonstrated for Reissner-Nordstrom black holes in four dimensions [1,2]. If one performs similar steps for this black hole then, before making use of the energy decomposition equations to fix the other constants, we can go back and compute the entropy for the extremal configuration since, for doing this, it is adequate to know the value of $T_{0}$. Then one sees that we need a finite value for $E_{0}$ to match everything consistently. With standard normalizations $T_{0}=1 / 8 \pi Q$ and $E_{0}=15 Q / 16$, where $Q$ is the ReissnerNordstrom charge. Making use of this funny shift in ground state energy, everything fits appropriately for extremal and near-extremal configurations. But for choosing a 'ground state', much like the $M=J=0$ state or the adS-state for the BTZ black hole, it is natural to pick up the state $M=Q=0$ for Reissner-Nordstrom black hole, which is the flat Minkowski space-time. This state should have zero temperature and zero entropy. In the gas model, $T=S=0$ implies that the ground state energy $E_{0}=0$. Now if one performs similar steps as for the BTZ case then one comes up with wrong prefactors in the entropy. Of course, the qualitative dependence on area always comes out, but we are not satisfied with this for a reasonable model. This certainly destroys the predictability of the improved approach advocated above. To our feeling, this is due to the wrong choice of the 'ground state', which for Reissner-Nordstrom is taken to be the Minkowski space. A correct choice would certainly furnish an improved gas model description of the Reissner-Nordstrom black hole. For the case

of the Schwarzschild black hole, however, such an extremal counterpart is missing and, hence, even a naive particle model computation of entropy is not possible. If, however, one attempts to build up the Schwarzschild black hole on the state $M=0, S=0, T=\infty$, then again the prefactors do not come out correctly. A detailed comparison between the gas model and the Schwarzschild black hole parameters reveals the fact that in this case too a funny ground state energy is needed.

\section{Entropy of Schwarzschild black hole}

In this section we consider the case of the Schwarzschild black hole. We will show how at least a naive gas model description can be provided in this case. The basic idea that we are going to use is the following:

One starts from a five-dimensional black string [7]:

$$
d s_{5}^{2}=-\left(1-\frac{2 m}{r}\right) d t^{2}+\left(1-\frac{2 m}{r}\right)^{-1} d r^{2}+r^{2} d \Omega_{2}+d y^{2}
$$

where $y$ represents a flat direction. When compactified in the flat direction using the 
standard Kaluza-Klein reduction, this provides a Schwarzschild black hole in four dimensions with mass $m$ and entropy $4 \pi m^{2}$, and we have set the four-dimensional Newton constant to unity. But if we first perform a Lorentz boost in the uncompactified $t-y$-plane and subsequently compactify the boosted $y$, then we get a charged dilatonic black hole in four dimensions. If the compactification radii in these two cases are related by $R_{1}=R_{2} \cosh \alpha$, where 1,2 refers to the Schwarzschild and charged black hole cases, respectively, and $\alpha$ is the boosting parameter, then the entropy of the non-extremal charged dilatonic black hole is identical to that of the Schwarzschild black hole [8]. Notice that $R_{2}$ is precisely the Lorentz contracted radius of $R_{1}$ under boosting. Certainly a boost along a compact internal dimension is not a symmetry of the solution but the entropy should be the same, since one expects that the number of states should not change under boosting [13,8]. Then it is reasonable to count the number of states for one classical configuration and predict the entropy of the other when two configurations are related to each other by boosting [9]. This is precisely what has been done in the following part of this section. A counting of states is performed for the fully non-extremal (we will not subject ourselves to a nearextremal limit in this case) charged dilatonic black hole to find out the entropy of the Schwarzschild black hole. One should note that for every compactification that gives rise to the Schwarzschild black hole there exists a corresponding compactification radius that gives rise to a charged dilatonic black hole and, hence, this identification makes sense. We will come back to the point of black string - black hole transition at the end. The problem of counting for these charged dilatonic black holes is that they usually have a singular BPS limit, in the sense that there is a curvature singularity at the horizon and the area of the horizon goes to zero in the extremal limit [14]. However, the thermodynamics of these black holes have been carried out in detail in the past $[15,16]$. Let us now provide a gas model counting of states for such black holes. We will use the convention used in $[17,16]$.

The generic black hole is $(G=1)$

$$
d s^{2}=-\frac{r^{2}-2 m r}{\sqrt{\Delta}} d t^{2}+\frac{\sqrt{\Delta}}{r^{2}-2 m r} d r^{2}+\sqrt{\Delta} d \Omega_{2},
$$

where $\Delta=r^{2}\left[r^{2}+2 m r\left(\cosh ^{2} \alpha-1\right)\right]$ and the dilaton is given by

$$
\phi=\ln \left(r^{2} / \sqrt{\Delta}\right)
$$

The ADM mass and the charge are given by

$$
M=\frac{1}{2} m\left(1+\cosh ^{2} \alpha\right)
$$




$$
Q=\frac{1}{\sqrt{2}} m \sinh \alpha \cosh \alpha .
$$

The Hawking temperature and entropy are given by (the horizon is at $r=2 m$ ):

$$
T_{H}^{-1}=8 \pi m \cosh \alpha, S_{H}=4 \pi m^{2} \cosh \alpha .
$$

The BPS limit of this black hole amounts to taking $m \rightarrow 0, \alpha \rightarrow \infty$, keeping $m \cosh ^{2} \alpha=m_{0}$ finite. In the extremal limit, $S_{H}=0, T_{H}=\infty, M=m_{0} / 2, Q=$ $m_{0} / \sqrt{2}$, which satisfies the BPS condition $M^{2}=Q^{2} / 2$.

Now comparing the gas model temperature, energy and entropy with those of the extremal black hole, we fix the following parameters:

$$
T=\infty, \quad N_{L}=N_{R}=0, \quad E_{0}=\frac{m_{0}}{2}
$$

Note that both the left and right temperatures need to be infinity in this case. Also the left and right sectors are behaving democratically in this case. There is a similarity with the $M \rightarrow 0$ limit of the Schwarzschild black hole, that the 'ground states' in both cases correspond to $S=0, T=\infty$. The important difference is that there is a finite ground state energy in this case, which we have already mentioned to be significant for describing the Schwarzschild black hole. Also note that for this extremal black hole $P=0$.

The non-extremal deformation is tuned by turning the parameter $m$ to a finite value. Let us subscribe to a specific case for which a gas model description can be obtained easily. So we choose a curve in the $(m, \alpha)$-plane, given by the equation $m \cosh ^{2} \alpha=m_{0}-m / 2$. This amounts to specifying a relation between the ADM mass and the charge. Note that this is a particular non-extremal deformation over the BPS solution (6.34) which is tuned by the parameter $m$. In the corresponding Schwarzschild case $m$ turns out to be the ADM mass. The ADM mass, temperature and entropy for this non-extremal black hole, when expressed in terms of the parameter $m_{0}$, are given by

$$
\begin{aligned}
M & =\frac{m_{0}}{2}+\frac{m}{4} \\
T_{H}^{-1} & =8 \pi \sqrt{m m_{0}-m^{2} / 2} \\
S_{H} & =4 \pi m \sqrt{m m_{0}-m^{2} / 2} .
\end{aligned}
$$

Also the charge becomes

$$
Q=\frac{m_{0}}{\sqrt{2}}\left(1-\frac{m}{2 m_{0}}\right)^{1 / 2}\left(1-\frac{3 m}{2 m_{0}}\right)^{1 / 2}
$$


and hence the BPS condition $M^{2}=Q^{2} / 2$ no longer holds. If we use the left-right democracy $N_{L}=N_{R}=N, T_{L}=T_{R}=T$ then it is possible to study the full nonextremal case rather than the near-extremal one. The number of parameters become half in this case, and the temperature and energy equations are sufficient to fix the rest of the parameters of the gas model. Equating the temperatures and ADM mass with the energy of the gas we get

$$
T_{H}=T, \quad L N=96 \pi m^{2}\left(m_{0}-m / 2\right) .
$$

So the gas-model entropy is given by $S=4 \pi m \sqrt{m m_{0}-m^{2} / 2}$, in perfect agreement with the non-extremal entropy of the charged dilatonic black hole, and hence, when boosted, with that of the Schwarzschild black hole. It is also instructive to calculate the central charge of the underlying CFT, as we did for the case of BTZ black hole. We choose the scale $L$, here, to be $m_{0}-m / 2$ characteristic to the curve we have chosen. Thus,

$$
c=96 \pi m^{2} .
$$

If one identifies the level from the definition (3.11) then (3.10) gives the desired expression for entropy. At this point one should note that with this central charge one can calculate the entropy for the Schwarzschild the black hole directly. Again we take a left-right symmetric gas with $T=1 / 8 \pi m$ in units where $G=1$. Also a direct extremal limit being absent the natural scale is set by the black hole mass, $L=m$. Then counting formula (3.12) gives rise to the entropy: $S=(\pi c L / 3) T=4 \pi m^{2}$, in agreement with that of the Schwarzschild black hole.

So we conclude that there exists a CFT description for the non-extremal charged dilatonic black holes, which have singular extremal limit. With the identification of the compactification radii these calculations indicate that there exists a CFT for the Schwarzschild black hole too for which we have calculated the central charge $c=96 \pi m^{2}$. At this point let me mention some subtleties involved in the identification of black hole entropies with two compactification radii. In the extremal limit the boosting is infinite and hence $R_{1} \gg R_{2}$. In the region where $R_{1}$ is larger than the Schwarzschild radius the black hole should be viewed as a black string and what we are computing is basically the entropy of the black string. So the extremal or nearextremal charged dilatonic configurations basically describe the back string entropy rather than the hole entropy. To really probe into the black hole region one should compute the entropy for configurations obtained by finite boostings. This forces us to consider the charged dilatonic black holes far from extremality. For a non-extremal 
charged dilatonic black hole, finite $\alpha$ and small $R_{2}$ imply small $R_{1}$ ( $R_{1}$ smaller than the Schwarzschild radius), which describes the black hole region appropriately.

\section{Acknowledgements}

The author would like to acknowledge discussions with Parthasarathi Mitra and Jnan Maharana. This research was supported in part by the World Laboratory.

Note: When this work was finished we saw a paper in the archive [18] also addressing the entropy of Schwarzschild black holes in four dimensions.

\section{References}

[1] S.P. de Alwis and K. Sato, Phys. Rev. D55 (1997) 6181, hep-th/9611189

[2] J. Maldacena and A. Strominger, Phys. Rev. D56 (1997) 4975, hep-th/9702015; see also R. Cai, Phys. Rev. Lett. 78 (1997) 2531, hep-th/9702142 for emergence of effective dimensionality to be one

[3] S. Carlip, Phys. Rev. D51 (1995) 632

[4] O. Coussaert and M. Henneaux, Phys. Rev. Lett. 72 (1994) 183; O. Coussaert, M. Henneaux and P. van Driel, Class. Quant. Grav. 12 (1995) 2961

[5] A. Strominger, hep-th/9712251

[6] I. Klebanov and L. Susskind, hep-th/9709108; E. Halyo, hep-th/9709225; L. Susskind, hep-th/9704080; T. Banks, W. Fischler, I. Klebanov and L. Susskind, hep-th/9709091; K. Sfetsos and K. Skenderis, hep-th/9711138

[7] J.H. Horne, G.T. Horowitz and A.R. Steif, Phys. Rev. Lett. 68 (1992) 568

[8] S.R. Das, S.D. Mathur, S.K. Rama and P. Ramadevi, hep-th/9711003

[9] F. Englert and E. Rabinovici, hep-th/9801048

[10] A. Strominger and C. Vafa, Phys. Lett. B379 (1996) 99, hep-th/9601029

[11] M. Banados, M. Henneaux, C. Teitelboim and J. Zanelli, Phys. Rev. D48 (1993) 1506 
[12] P. Mitra, hep-th/9712252

[13] G.T. Horowitz and E. Martinec, hep-th/9710217

[14] S.S. Gubser, I.R. Klebanov and A.W. Peet, Phys. Rev. D54 (1996) 3915, hepth/9602135

[15] R. Kallosh, A. Linde, T. Ortin, A. Peet and A. van Proyen, Phys. Rev. D46 (1992) 5278; R. Kallosh, Phys. Lett. B282 (1992) 80; A. Ghosh and J. Maharana, Mod. Phys. Lett. A11 (1996) 3103

[16] A. Ghosh and P. Mitra, hep-th/9509090

[17] A. Sen, Mod. Phys. Lett. A10 (1995) 2081, hep-th/9504147

[18] R. Arguiro, F. Englert and L. Houart, hep-th/9801053 